cancers, diabetes and cardiovascular disease in a society with rapidly changing life styles, increasing sedentarity and westernized diet. The main exposures of interest are dietary intake, physical activity, obesity and environmental exposure in particular endocrine disruptor chemicals as well as gene by environment interaction. The study started in 2006 enrolling teachers from 2 states (Veracruz and Jalisco) in 2008-2009, 10 others states were included. To date the study enrolled close to 116000 women. Overall the response rate has been $64 \%$, and follow-up at 4 years $71 \%$. Information on sociodemographic, lifestyle, reproductive history, medical history, anthropometry, physical activity and diet is collected through biennial questionnaire. In addition in a subsample of the study population detailed information including mammographic density, bone density, blood pressure, spirometry, impedance and anthropometric measurements, blood and urinary samples as well as more detailed questionnaire on lifestyle. This large cohort with a high variability in lifestyle and fast changing habits will provide very valuable information on risk factors of chronic diseases in female.

\section{1-6.3 LIFE STYLE FACTORS \& BODY MASS INDEX AS PREDICTOR OF MORTALITY: FINDINGS FROM A COHORT STUDY IN INDIA}

doi:10.1136/jech.2011.142976a.44

\section{K Ramadas. * Regional Cancer Centre, Trivandrum, India}

A population based cohort study including 167343 adults aged 34 and above living in a rural area were recruited and followed-up during January 1996-December 2004 as part of an ongoing oral cancer screening programme. Blood pressure and body mass index (BMI) were measured at baseline and at 3 year intervals. Demographic details and lifestyle factors were also collected through a questionnaire. These subjects were actively followed-up for vital status by field workers through municipal death registers, records collected from social and religious organisations, and during repeated house visits and cause of deaths were determined by verbal autopsy.

The mortality risks associated with chewing (and 95\% CIs), after adjusting for age, sex, socio-economic dietary variables, and for other habits, were 0.90 (0.86 to 0.94) for all-cause, 1.07 (0.94 to 1.22$)$ for cancer and 1.22 (1.04 to 1.44$)$ for tobacco-related cancer; with smoking the respective mortality risks were 1.31 (1.24 to 1.39 ), 1.63 (1.37 to 1.94$)$ and 1.68 (1.36 to 2.08); and with alcohol use the risks were 1.13 (1.06 to 1.20), 1.32 (1.11 to 1.57 ) and 1.47 (1.19 to 1.80 ). Reduced risk of all-cause mortality by chewing was observed only in the 60-84 years old group $(0.90$ (0.85 to 0.94$)$ ), and detrimental effects of chewing on cancer mortality were shown in the young and middle-age groups: $34-39$ years old (1.33 (0.67 to 2.65)), and $40-59$ years old (1.26 (1.03 to 1.55$)$ ). An analysis of the association between BMI and change in weight in relation to mortality risk showed that low BMI was found to be a predictor of mortality, while high BMI was not.

\section{1-6.4 PARTNERSHIP FOR COHORT RESEARCH AND TRAINING (PaCT): PILOT STUDY, SOUTH AFRICA}

doi:10.1136/jech.2011.142976a.45

J Volmink.* Stellenbosch University, Stellenbosch, South Africa

An epidemic of chronic, non-communicable diseases is growing in Africa. By 2030, according to the WHO, three of the top four causes of death in low-income countries will be heart disease, stroke, and chronic lung disease. No large scale epidemiologic studies investigating chronic diseases have been conducted in Africa.

PaCT, a component of the Global Epidemiology Initiative established at the Harvard School of Public Health, is an ambitious project which will investigate chronic, non-communicable diseases among 500000 people from four African countries over the next 20 years. This initiative will also provide training opportunities for African researchers. PaCT includes scientists from universities in South Africa, Uganda, Tanzania, Nigeria, as well as Harvard University. Pilot studies are being conducted in all four of these countries.

An initial cross-sectional baseline survey, with 6 month longitudinal follow-up was planned. We aimed to recruit 800-1000 school teachers employed at public schools within the CapeTown metropolitan area. After returning a self-administered questionnaire and completed consent forms, teachers were visited at the schools by trained nurses who took physical measurements (weight, height, waist circumference and blood pressure) and collected biological (blood and urine) samples.

Our presentation will provide a brief overview of PaCT activities in the four African countries involved. It will then focus on practical challenges encountered in the pilot studies and how they were addressed, with special reference to the South African pilot study. These challenges include funding procurement, standardisation of methods across countries, obtaining approval for the study, participant recruitment, data collection and analysis. 